\title{
Interrelationship of Yield and Quality Attributing Traits in JNPT Lines of Rice
}

\author{
Pratibha Choudhary ${ }^{1 *}$, D. K. Mishra ${ }^{1}$, G.K. Koutu ${ }^{1}$, Amita Pachori ${ }^{2}$ and S. K. Singh ${ }^{1}$ \\ ${ }^{1}$ Dept. of Plant Breeding and Genetics, Jawaharlal Nehru Krishi Vishwa Vidyalaya, Jabalpur, M.P. (482 004), India \\ ${ }^{2}$ Dept. of Plant Pathology, Rajmata Vijayaraje Scindia Krishi Vishwa Vidyalaya, Gwalior, M.P. (474 002), India
}

\author{
Corresponding Author \\ Pratibha Choudhary \\ e-mail: prratibha08@gmail.com
}

\author{
Article History \\ Article ID: 3 C0934 \\ Received in 02 ${ }^{\text {nd }}$ November, 2017 \\ Received in revised form $19^{\text {th }}$ April, 2018 \\ Accepted in final form 21 $1^{\text {st }}$ May, 2018
}

\begin{abstract}
In present study, one hundred eighty five JNPT (Jawahar New Plant Type) lines including five checks were evaluated for twenty eight morphological and quality traits planted in Randomized Complete Block Design with three replications. The experiment was conducted during kharif seasons of 2014 and 2015 at Seed Breeding Farm, Department of Plant Breeding and Genetics, College of Agriculture, JNKVV, Jabalpur (M.P.), India. Observations were recorded on the basis of middle five random competitive plants selected from each line in every replication for yield and quality traits. Considerable genetic variability was exhibited by all the yield and quality traits under study. It was observed that for selecting the high yielding lines in the rice the characters viz., spikelet density, fertile spikelets panicle ${ }^{-1}$, number of spikelets per panicle, panicle weight plant ${ }^{-1}$ and biological yield plant ${ }^{-1}$ might be considered. On the basis of high PC score in principal component analysis 10 most prominent JNPT lines JNPT 813, JNPT 811, JNPT 845, JNPT 770, JNPT 779, JNPT 777, JNPT 778, JNPT 749, JNPT 781 and JNPT(S) $10 \mathrm{H}$ were identified. Thus, these JNPT lines will be utilized as inbred for production of hybrid rice, with higher yield and better quality. However, after evaluation under different agro-ecological rice growing situations, these lines might be released as high yielding variety with better quality.
\end{abstract}

Keywords: Rice, JNPT lines, PC score, PCA, variability

\section{Introduction}

Rice (Oryza sativa L.) is the basic food crop of Asia, providing over $30 \%$ of the calories consumed in the region (Narciso and Hossain, 2002). Rice production in Asia has increased by 2.6 times since 1961, primarily as a result of the "Green Revolution", which dramatically increased the rice productivity in the high-input irrigated systems (Khush, 1999). Enhancing crop yield is one of the top most priorities in crop breeding programmes. Results have indicated that an effective way to develop super rice lies first in developing the new plant type and strong vigour by crossing Indica with Japonica subspecies, and then consolidating the two advantages by optimizing the combination of desirable traits via multiple crossing and backcrossing (Cheng et al., 2001). In the late 1980s, to increase yield potential in Indica inbred varieties under a tropical environment, a breeding programme to develop new plant type (NPT) rice was launched at IRRI. The NPTlines had several traits from tropical Japonica: low tillering habit, few unproductive tillers, large panicles, thick culm, lodgingresistance, and large and dark green flag leaves (Khush, 1995). Especially, the NPT lines had elite characteristics such as larger flag leaves, higher spikelet number, and heavier grain weight than IR64. Thus, these lines were thought to be useful materials to improve the yield potential of IRRI-bred varieties, including IR64. Jawaharlal Nehru Krishi Vishwa Vidyalaya, Jabalpur (India) has made JNPT (Jawahar New Plant Type) lines through tropical Japonicax/ndica hybridization using wide compatibility gene source. The Indica lines used to develop the JNPT lines were very popular for high yield and quality aspects. Derived lines combine strong culm, short stature, dark green erect leaves, long panicles, high grain numbers with improved quality.

Magnitude of genetic variability present in the plant population decides the efficiency of selection as well as development of an effective plant breeding strategy. Correlations help the breeder to understand the mutual component characters on which selection can be based for genetic improvement (Chakravorty et al., 2013). Information on association of the characters, direct and indirect effects contributed by each trait towards yield will be an added advantage in aiding the selection process and helps the breeder to design his selection strategies for improving the grain yield (Ravindrababu et al., 2012). Principal Component Analysis (PCA) is one of the tools available for summarizing and describing the inherent 
genetic variation in crop genotypes. This technique helps in identification of traits that help in distinguishing selected genotypes based on similarities in one or more traits and classify the genotypes into separate groups (Ariyo, 1987 and Nair et al., 1998). The PCA has been used by Nassir, 2002 and Chakravorty et al., 2013 in rice for partitioning observed variation and studying inter relationships among different traits. PCA helps to identify the traits with high variability, correlations reveal the strength of relationship between different traits with yield. Thus, to identify the yield and quality traits of utmost importance the present investigation was conducted in rice by studying genetic parameters and interrelationship of yield and quality traits in JNPT lines.

\section{Materials and Methods}

The experimental material consists of 180 JNPT lines derived from Indicax Japonica subspecies crosses ( $F_{14}-F_{15}$ generation's) developed by JNKVV, Jabalpur with 5 checks were grown during Kharif seasons of 2014 and 2015 at Seed Breeding Farm, JNKVV, Jabalpur (M.P.), India. These lines were planted in Randomized Complete Block Design with three replications. Twenty one days old seedlings were transplanted in the experimental site with spacing of $20 \mathrm{~cm}$ between plant to plant and $30 \mathrm{~cm}$ between the rows, keeping single seedling per hill. Gap filling was done within a week in order to maintain uniform plant population. Fertilizer dose of $120 \mathrm{~kg}$ $\mathrm{N}, 60 \mathrm{~kg} \mathrm{P}_{2} \mathrm{O}_{5}$ and $60 \mathrm{~kg} \mathrm{~K} \mathrm{O}_{2}$ was applied. Observations were recorded on the basis of middle five random competitive plants selected from each line in every replication for yield and quality traits. The data for each trait was statistically analyzed using analysis of variance recommended for randomized complete block design. The mean values were used to obtain analysis of variance as per methodology advocated by Panse and Sukhatme (1967). PCV and GCV were calculated by the formula given by Burton (1952), heritability in broad sense by Burton (1952) and Burton and De Vane (1953) and genetic advance i.e. the expected genetic gain was calculated by using the procedure given by Johnson et al., 1955. Correlation coefficient and path coefficient analysis was worked out as method suggested by Al-Jibouri et al., 1958 and Dewey and Lu (1959) respectively. Genetic and phenotypic correlations among the traits were determined by Singh and Chaudhary (2005) method. The simplified procedure of (Juliano, 1971) is used for the amylose contentanalysis. PCA analysis was done using the methodology given by (Massy, 1965; Jolliffe, 1986).

\section{Results and Discussion}

\subsection{Genetic parameter}

The mean value of different characters were tested for homogeneity by Bartlett's test and found non-significant. Thus, the pooled result of two years was obtained by computing the mean value of different traits from two years. All yield attributing traits showed the considerable amount of variability. The total number of spikelets panicle ${ }^{-1}$ (34420.0178) showed maximum variability, whereas, stem thickness (0.0674) exhibited minimum variability, which was in agreement with the findings of Kumar et al. (2015). Highest genotypic and phenotypic coefficient of variation observed by spikelet density (34.77 and 34.91) while, low GCV and PCV by hulling percentage (4.53 and 4.59). High heritability coupled with high genetic advance expressed by spikelet density followed by fertile spikelets panicle ${ }^{-1}$, number of spikelets panicle $^{-1}$, number of productive tillers plant ${ }^{-1}$, panicle weight per plant, amylose content, number of tillers plant ${ }^{-1}$, grain yield plant ${ }^{-1}$, biological yield plant ${ }^{-1}, 1000$-grain weight, flag leaf width, harvest index, flag leaf length, panicle index, stem length, grain length, plant height, grain breadth, decorticated grain $\mathrm{l} / \mathrm{b}$ ratio, panicle length and decorticated grain length (Table 1). This was in consonance with the findings of Bekele et al. (2013), Rajput et al. (2014); Shrivastava et al. (2014) and Dongre et al. (2014).

\subsection{Character association}

Grain yield plant ${ }^{-1}$ revealed significant and positive association with panicle weight plant ${ }^{-1}$, biological yield per plant, number of productive tillers plant ${ }^{-1}$, harvest index, number of tillers plant $^{-1}$, fertile spikelets per panicle, spikelet density, number of spikelets panicle ${ }^{-1}$, flag leaf length, panicle index, days to $50 \%$ flowering, grain breadth, days to maturity, spikelet fertility, hulling percentage and amylose content in continues two years and pooled result of two years. Similar findings were reported by Sohgaura et al. (2014), Singh et al. (2014), Dongre et al. (2014); Shrivastava et al. (2014). Considering the results from correlation and path coefficient analysis, it is concluded that for selecting the high yielding lines in rice the characters viz., panicle weight plant ${ }^{-1}$, panicle index, biological yield per plant, harvest index, number of productive tiller plant $^{-1}$, spikelet density, panicle length, spikelet fertility and 1000-grain weight might be considered (Table 2(a), 2(b), 3(a) \& 3(b)).

\subsection{Principal component analysis}

To find out independent impact of all the characters under study principal component analysis was conducted. In JNPT lines, the first principal component accounted for maximum proportion of total variability in the set of all variables and remaining components accounted for progressively lesser and lesser amount of variation. Out of 28 principal components (PCs) only eight PCs exhibited more than 1.00 Eigen value and about $\mathbf{7 5 . 6 1 \%}$ variability among the traits studied (Table 4). So, these eight PC's were given due importance for further explanation. Highest variability $(20.69 \%)$ was exhibited by PC1 however, PC2, PC3, PC4, PC5, PC6, PC7 and PC8 revealed $14.20 \%, 9.89 \%, 8.90 \%, 6.45 \%, 5.62 \%, 5.30 \%$ and $4.54 \%$ variability, respectively among the lines for the traits under 


\begin{tabular}{|c|c|c|c|c|c|c|c|c|c|}
\hline \multirow{2}{*}{$\begin{array}{l}\text { SI. } \\
\text { No. }\end{array}$} & \multirow[t]{2}{*}{ Traits } & \multirow[t]{2}{*}{ Mean } & \multicolumn{2}{|c|}{ Range } & \multirow{2}{*}{$\begin{array}{c}\text { GCV } \\
(\%)\end{array}$} & \multirow{2}{*}{$\begin{array}{l}\text { PCV } \\
(\%)\end{array}$} & \multirow{2}{*}{$\begin{array}{l}h^{2} \text { (Broad } \\
\text { Sense \%) }\end{array}$} & \multirow{2}{*}{$\begin{array}{l}\text { Genetic } \\
\text { Advance }\end{array}$} & \multirow{2}{*}{$\begin{array}{l}\text { Gen. Adv as } \\
\% \text { of mean }\end{array}$} \\
\hline & & & Mini. & Maxi. & & & & & \\
\hline 1. & DFF & 103.60 & 84.11 & 131.91 & 8.81 & 8.82 & 99.7 & 18.788 & 18.136 \\
\hline 2. & DTM & 129.91 & 108.72 & 157.34 & 7.03 & 7.04 & 99.6 & 18.788 & 14.462 \\
\hline 3. & FLL & 40.30 & 20.63 & 60.36 & 19.59 & 19.645 & 99.4 & 16.22 & 40.244 \\
\hline 4. & FLW & 1.99 & 0.87 & 2.98 & 19.74 & 19.81 & 99.2 & 0.807 & 40.512 \\
\hline 5. & ST & 1.94 & 1.57 & 2.37 & 7.69 & 7.82 & 96.7 & 0.302 & 15.575 \\
\hline 6. & SL & 94.04 & 62.17 & 141.00 & 17.07 & 18.87 & 90.46 & 32.996 & 35.089 \\
\hline 7. & $\mathrm{PH}$ & 121.96 & 87.64 & 179.79 & 14.76 & 14.98 & 98.53 & 37.05 & 30.381 \\
\hline 8. & PL & 27.92 & 19.77 & 40.58 & 13.23 & 13.47 & 96.5 & 7.476 & 26.778 \\
\hline 9. & TPP & 6.99 & 3.50 & 15.44 & 27.54 & 27.66 & 99.1 & 3.946 & 56.493 \\
\hline 10. & PTPP & 6.46 & 3.15 & 13.52 & 28.48 & 28.7 & 98.5 & 3.764 & 58.232 \\
\hline 11. & BYPP & 74.13 & 24.82 & 120.36 & 23.22 & 23.97 & 96.87 & 35.39 & 47.73 \\
\hline 12. & PWPP & 29.67 & 12.05 & 60.57 & 28.56 & 29.71 & 96.12 & 17.44 & 58.80 \\
\hline 13. & FSPP & 276.36 & 104.78 & 567.99 & 32.41 & 32.99 & 98.24 & 186.22 & 67.38 \\
\hline 14. & TSPP & 334.62 & 138.68 & 708.59 & 32.01 & 32.82 & 97.53 & 220.65 & 65.94 \\
\hline 15. & SF & 82.91 & 56.45 & 94.85 & 9.84 & 9.86 & 99.8 & 16.79 & 20.26 \\
\hline 16. & SD & 12.20 & 5.45 & 25.69 & 34.77 & 34.91 & 99.2 & 8.70 & 71.34 \\
\hline 17. & TGW & 24.97 & 11.59 & 36.62 & 20.86 & 20.87 & 99.9 & 10.73 & 42.96 \\
\hline 18. & $\mathrm{HI}$ & 32.88 & 17.61 & 49.26 & 20.32 & 20.50 & 98.3 & 13.64 & 41.49 \\
\hline 19. & $\mathrm{PI}$ & 82.67 & 44.35 & 132.42 & 18.29 & 18.46 & 98.2 & 30.87 & 37.34 \\
\hline 20. & $\mathrm{GL}$ & 8.80 & 5.46 & 12.51 & 15.03 & 15.06 & 99.5 & 2.72 & 30.88 \\
\hline 21. & $\mathrm{~GB}$ & 2.65 & 2.18 & 4.05 & 12.82 & 12.84 & 99.7 & 0.70 & 26.37 \\
\hline 22. & $\mathrm{HP}$ & 77.80 & 60.21 & 85.57 & 4.53 & 4.59 & 98.69 & 7.26 & 9.33 \\
\hline 23. & $\mathrm{MP}$ & 66.83 & 30.88 & 77.54 & 9.12 & 9.39 & 97.12 & 12.54 & 18.77 \\
\hline 24. & DGL & 5.45 & 4.06 & 7.57 & 11.08 & 11.11 & 99.6 & 1.24 & 22.79 \\
\hline 25. & DGB & 2.22 & 1.83 & 3.05 & 7.78 & 7.85 & 98.3 & 0.35 & 15.89 \\
\hline 26. & DLBR & 2.47 & 1.66 & 3.71 & 13.02 & 13.10 & 98.9 & 0.66 & 26.68 \\
\hline 27. & AP & 18.72 & 11.33 & 31.35 & 27.29 & 27.69 & 98.5 & 10.52 & 56.19 \\
\hline 28. & GYPP & 23.96 & 11.92 & 45.57 & 26.22 & 26.58 & 98.64 & 12.91 & 53.86 \\
\hline
\end{tabular}

study. Rotated component matrix revealed that each principal component separately loaded with various yield and quality attributing traits under study (Table 5). The PC1 was more related to the yield attributing traits viz., spikelet density, fertile spikelets per panicle and number of spikelets per panicle. PC2 exhibited positive effect for grain yield per plant, panicle weight per plant and biological yield plant ${ }^{-1}$, which were more loaded with yield contributing traits. Thus, PC1 and PC2 allowed for simultaneous selection of yield related traits and it can be regarded as yield factor. The $5^{\text {th }}$ principal component was more linked to quality attributing traits i.e., decorticated grain length and decorticated grain $\mathrm{I} / \mathrm{b}$ ratio. Similarly, eighth PC also more dominated with quality traits such as hulling percentage and milling percentage. Remaining principal components (PC3, PC4, PC6 and PC7) more loaded with physiological traits (Table 6). This result was in agreement with Yang et al. (2009), Ashfaq et al. (2012) and Kumar et al. (2014). On the basis of PCA study, it was cleared that the JNPT 810, JNPT 754, JNPT 800, JNPT 752, JNPT 811, JNPT 751, JNPT 748, JNPT 820, JNPT 822 and JNPT 830 were the selected 10 promising lines for both yield and quality attributes. Because, these genotypes performing their presence with high PC score in both yield (PC1or PC2) as well as quality (PC5 or PC8) related PC's. Therefore, selection of genotype in these PCs will be more accurate in comparison to other PCs. (Table 7). 


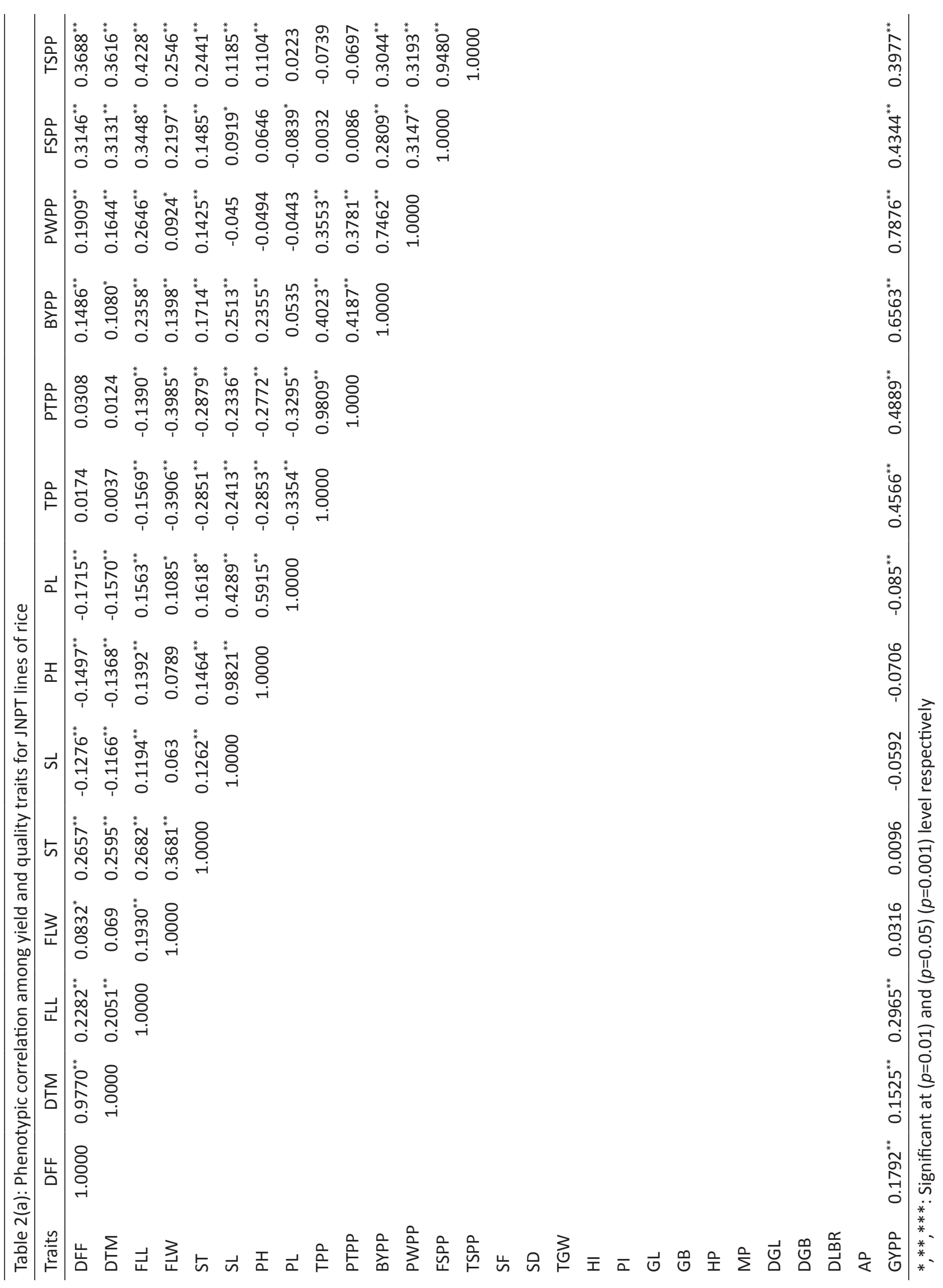




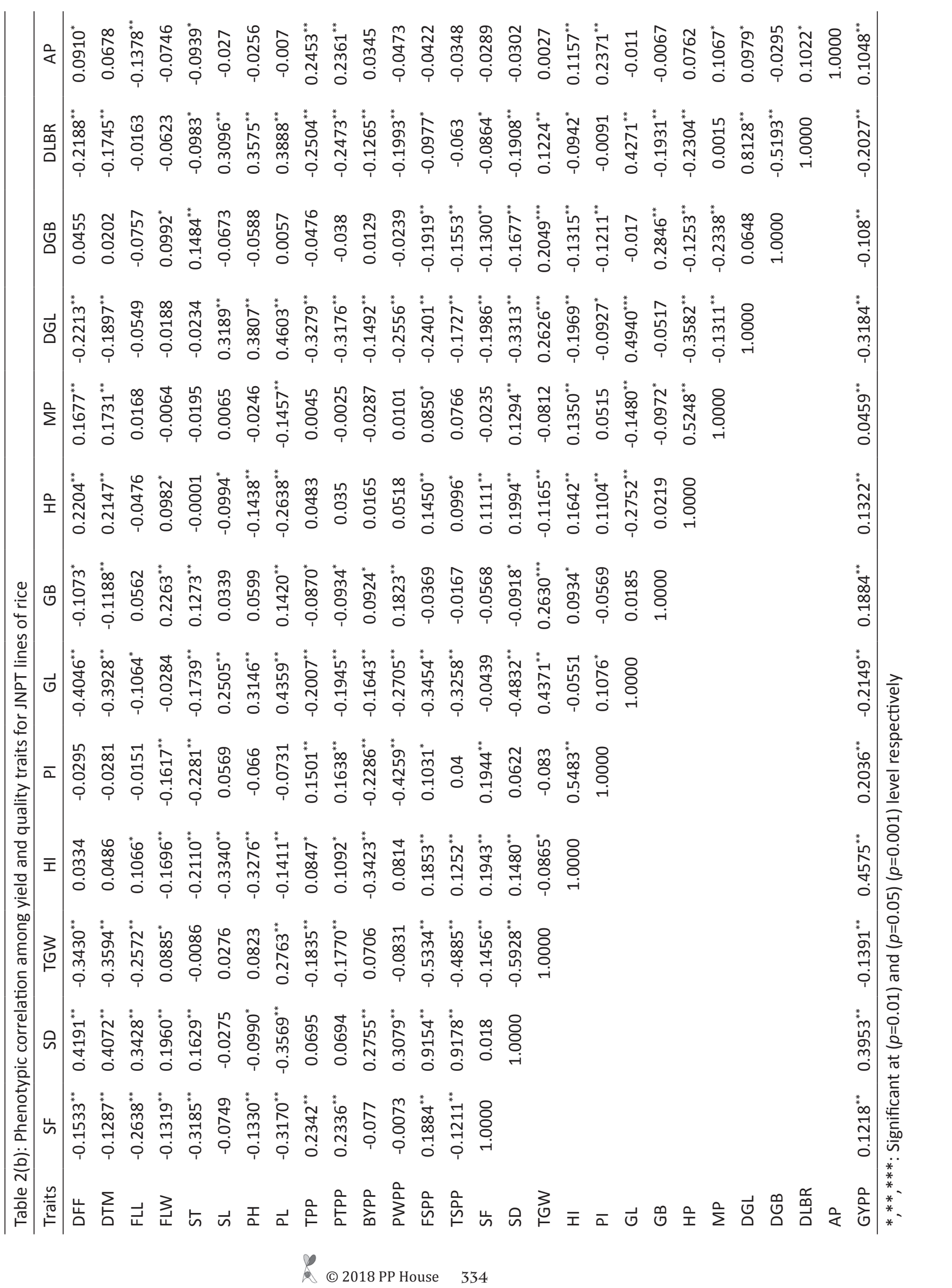




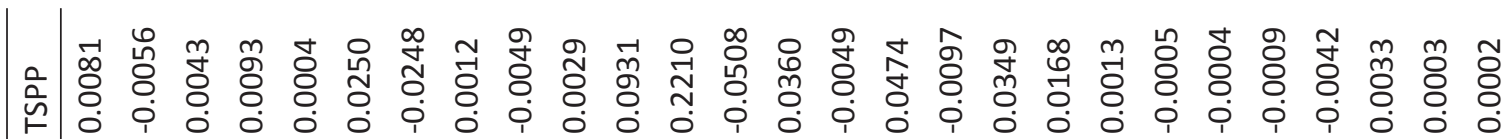

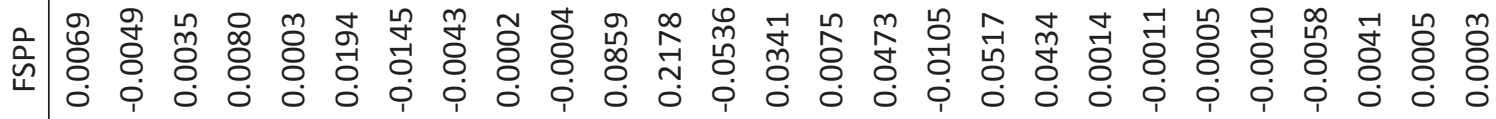

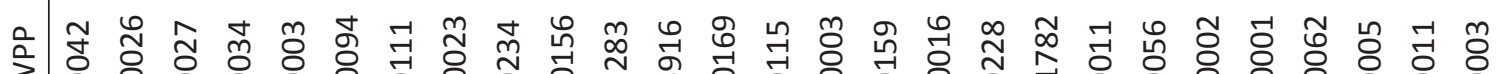

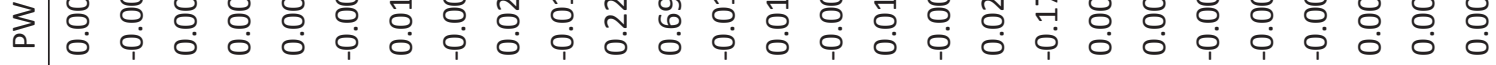

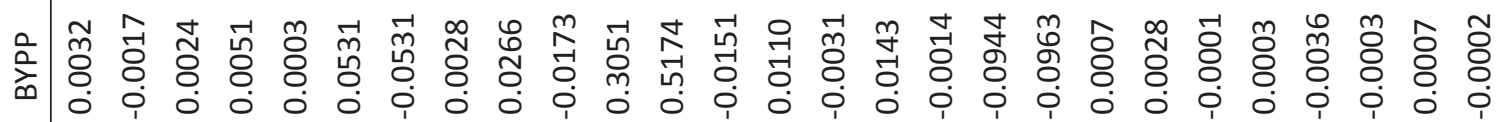

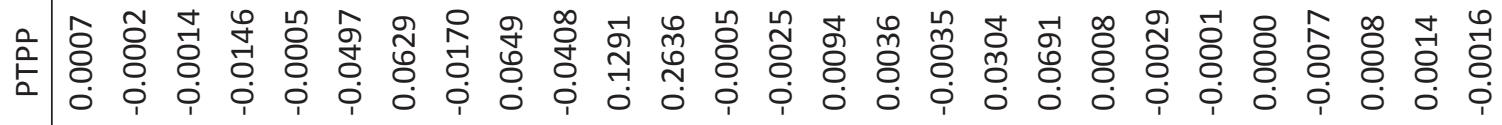

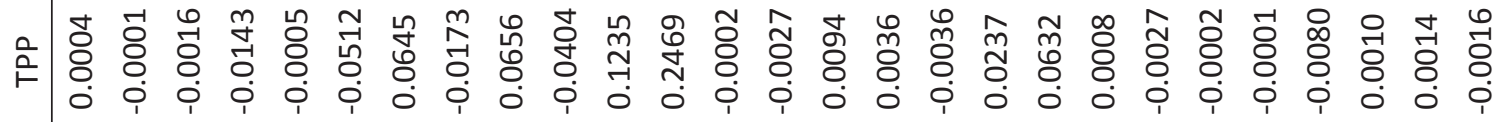

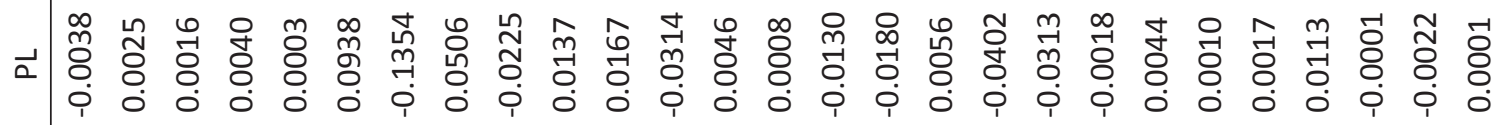

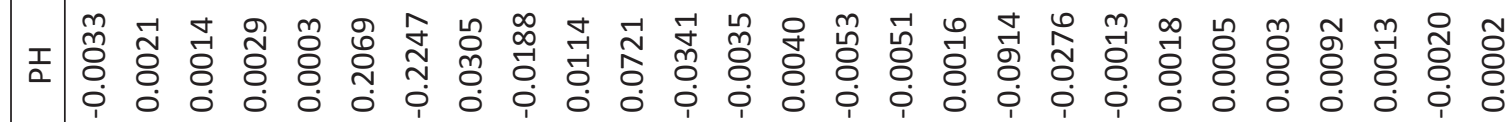
$\stackrel{9}{.0}$

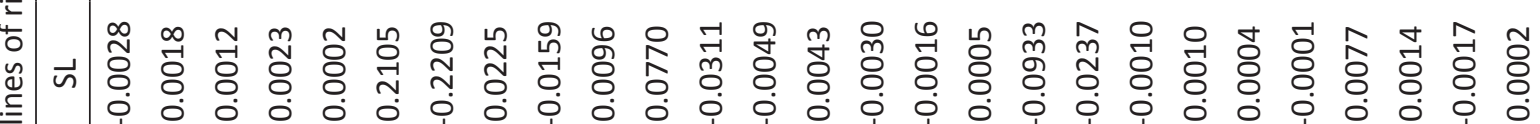
号 ๘)

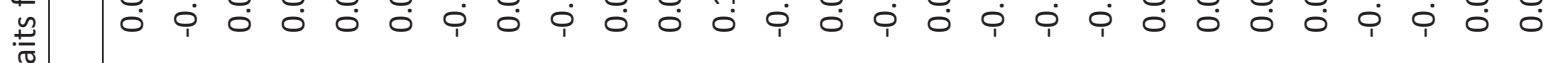

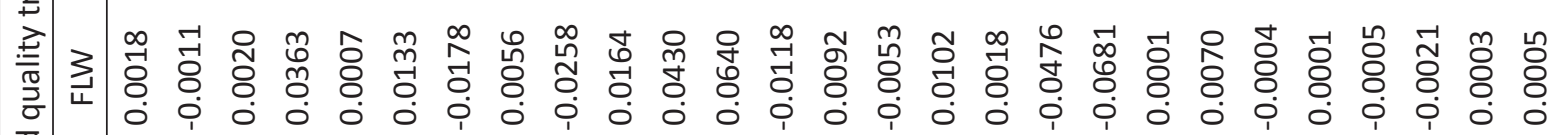
$\frac{\bar{c}}{\pi}$

光 \begin{tabular}{llllllllllllllllll}
0 & 0 \\
\hline
\end{tabular} 点

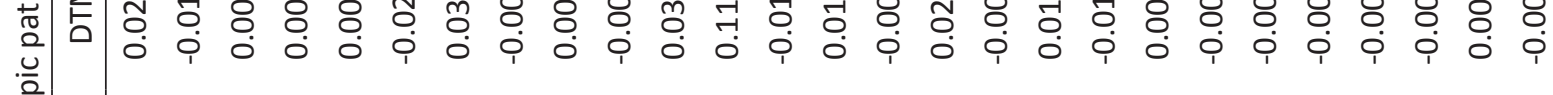
$\sum_{\frac{2}{2}}^{\frac{0}{2}}$

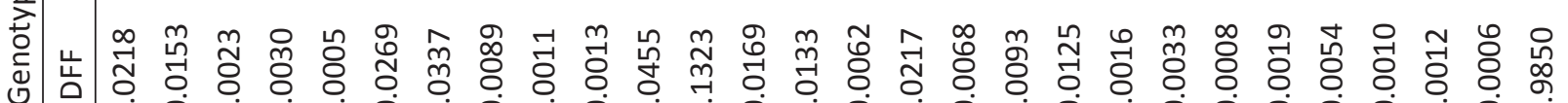

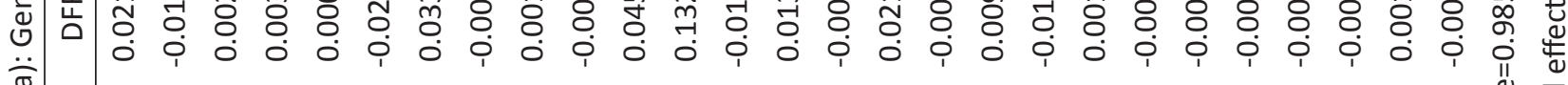

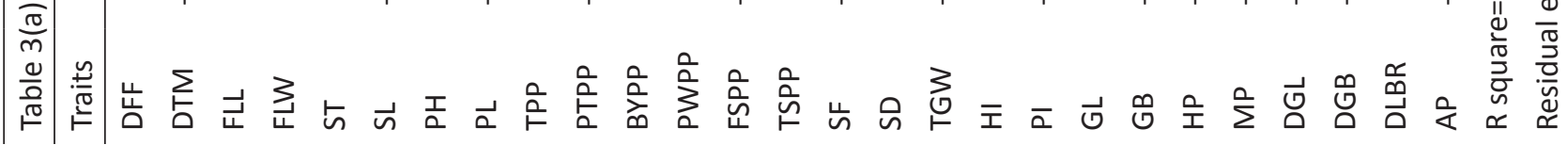




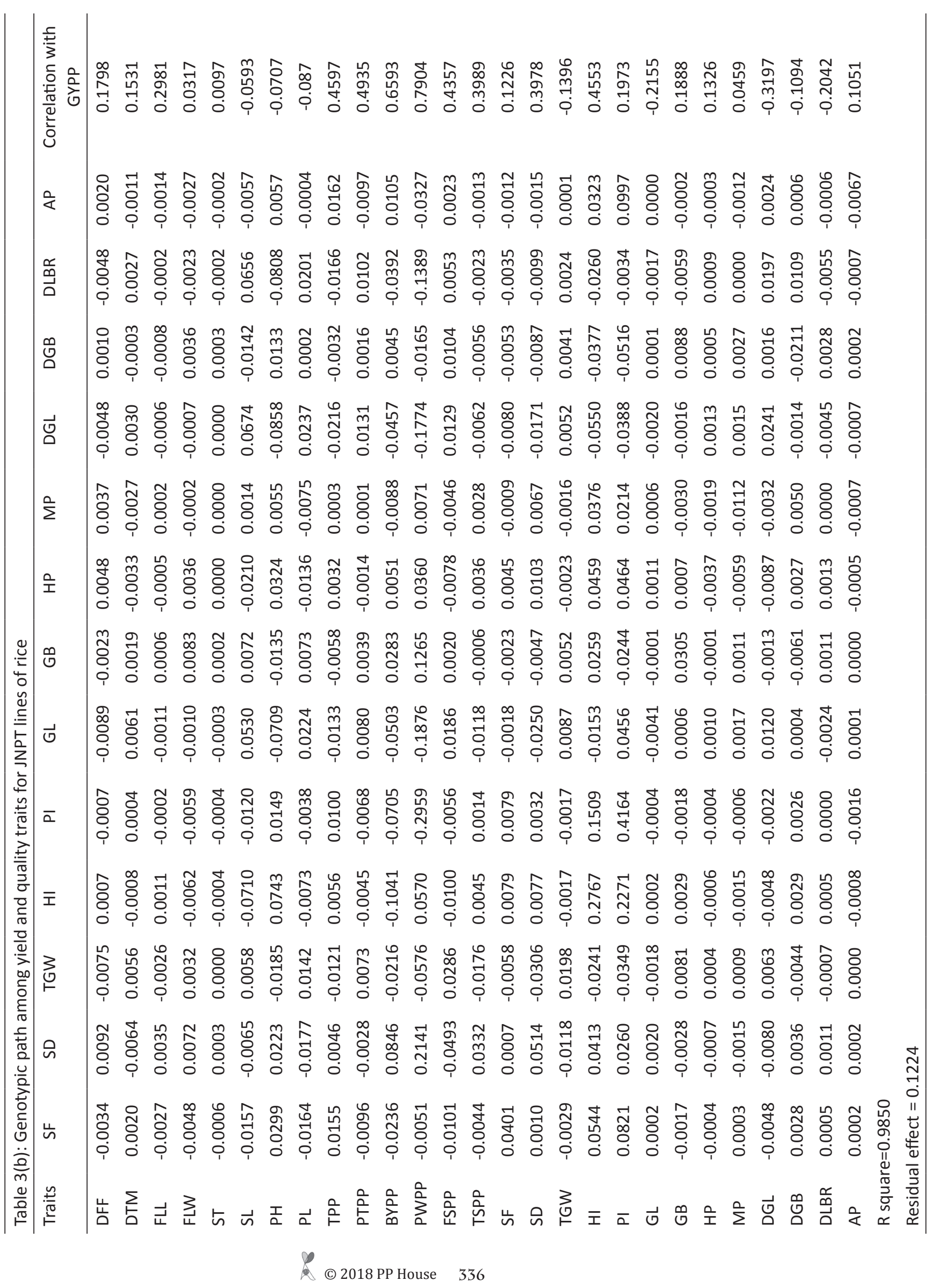




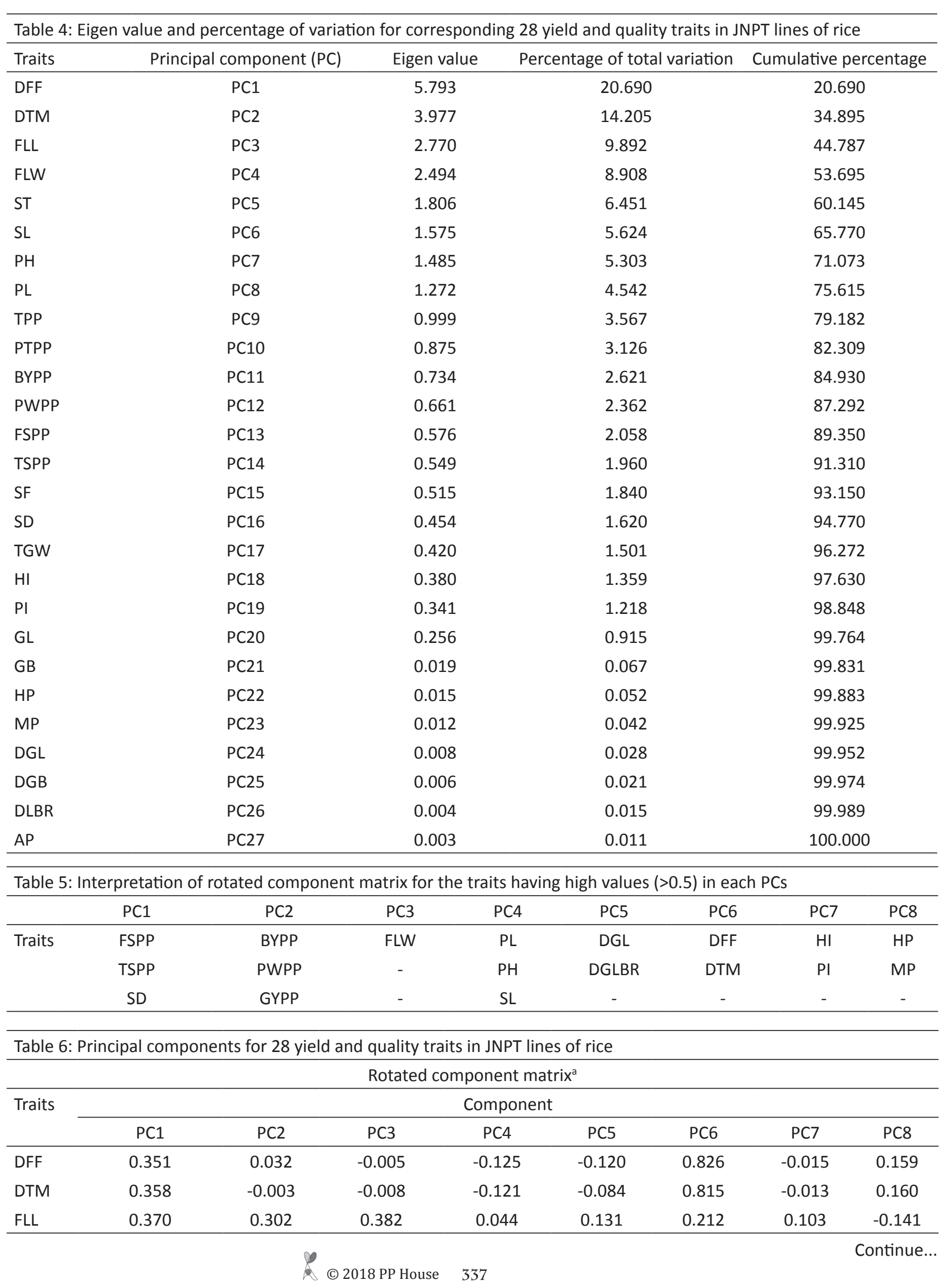




\begin{tabular}{|c|c|c|c|c|c|c|c|c|}
\hline \multicolumn{9}{|c|}{ Rotated Component Matrixa } \\
\hline \multirow[t]{2}{*}{ Traits } & \multicolumn{8}{|c|}{ Component } \\
\hline & PC1 & PC2 & PC3 & PC4 & PC5 & PC6 & PC7 & PC8 \\
\hline FLW & 0.112 & 0.103 & 0.655 & 0.062 & -0.147 & 0.008 & -0.117 & 0.101 \\
\hline ST & 0.102 & 0.110 & 0.497 & 0.121 & -0.165 & 0.379 & -0.226 & -0.031 \\
\hline SL & 0.083 & -0.007 & 0.069 & 0.938 & 0.077 & -0.077 & -0.114 & 0.027 \\
\hline $\mathrm{PH}$ & 0.021 & 0.012 & 0.133 & 0.950 & 0.128 & -0.054 & -0.080 & -0.031 \\
\hline PL & -0.258 & 0.086 & 0.349 & 0.553 & 0.286 & 0.072 & 0.105 & -0.265 \\
\hline TPP & 0.006 & 0.470 & -0.789 & -0.157 & -0.162 & -0.014 & 0.051 & 0.009 \\
\hline PTPP & 0.008 & 0.491 & -0.787 & -0.154 & -0.155 & -0.005 & 0.071 & -0.007 \\
\hline BYPP & 0.163 & 0.806 & -0.133 & 0.295 & -0.110 & 0.096 & -0.284 & 0.001 \\
\hline PWPP & 0.164 & 0.909 & 0.029 & -0.113 & -0.032 & 0.052 & -0.186 & 0.026 \\
\hline FSPP & 0.903 & 0.214 & 0.140 & 0.081 & -0.008 & -0.007 & 0.136 & 0.051 \\
\hline TSPP & 0.846 & 0.241 & 0.253 & 0.109 & 0.043 & 0.157 & 0.104 & -0.009 \\
\hline SF & 0.207 & -0.090 & -0.360 & -0.081 & -0.142 & -0.517 & 0.110 & 0.146 \\
\hline SD & 0.905 & 0.185 & 0.082 & -0.084 & -0.057 & 0.126 & 0.037 & 0.101 \\
\hline TGW & -0.752 & 0.087 & 0.264 & 0.065 & -0.003 & -0.147 & 0.051 & -0.033 \\
\hline $\mathrm{HI}$ & 0.126 & 0.093 & 0.030 & -0.377 & 0.063 & -0.090 & 0.805 & 0.099 \\
\hline $\mathrm{PI}$ & 0.122 & -0.216 & -0.241 & 0.065 & -0.054 & -0.062 & 0.815 & 0.048 \\
\hline $\mathrm{GL}$ & -0.452 & -0.110 & 0.094 & 0.315 & 0.338 & -0.238 & 0.195 & -0.242 \\
\hline GB & -0.245 & 0.308 & 0.415 & 0.086 & -0.407 & -0.118 & 0.236 & -0.038 \\
\hline HP & 0.084 & 0.021 & 0.036 & -0.075 & -0.168 & 0.051 & 0.093 & 0.838 \\
\hline MP & 0.012 & 0.008 & 0.013 & -0.001 & 0.160 & 0.129 & 0.054 & 0.819 \\
\hline DGL & -0.316 & -0.182 & 0.124 & 0.356 & 0.574 & 0.091 & 0.000 & -0.338 \\
\hline DGB & -0.267 & -0.060 & 0.131 & 0.044 & -0.690 & 0.189 & -0.029 & -0.340 \\
\hline DLBR & -0.124 & -0.113 & 0.034 & 0.279 & 0.880 & -0.037 & 0.017 & -0.097 \\
\hline AP & -0.167 & 0.064 & -0.332 & 0.152 & 0.047 & 0.339 & 0.383 & 0.123 \\
\hline GYPP & 0.274 & 0.845 & -0.090 & -0.056 & -0.066 & 0.002 & 0.353 & 0.063 \\
\hline
\end{tabular}

Extraction Method: Principal Component Analysis. Rotation Method: Varimax with Kaiser Normalization

Table 7: Selected JNPT lines of rice on the basis of high PC score for pooled data of two years

\begin{tabular}{|c|c|c|c|c|c|c|c|}
\hline PC1 & PC2 & PC3 & PC4 & PC5 & PC6 & PC7 & PC8 \\
\hline JNPT 814 & JNPT 35-01 & JNPT 753 & JNPT (S) 10-1 C & JNPT 769 & JNPT 780 & JNPT 759 & JNPT 754 \\
\hline JNPT 813 & JNPT 37-01 A & JNPT 806 & JNPT 771 & JNPT (S) 10-1 C & JNPT 781 & PUSA BASMATI 1 & JNPT 792 \\
\hline JNPT 816 & JNPT-24 & JNPT 765 & JNPT (S) $10 \mathrm{E}$ & Sahbhagi & JNPT-47 & JNPT 851 & JNPT 776 \\
\hline JNPT 810 & JNPT 32-01 & JNPT 838 & JNPT (S) 10-1 B & JNPT 849 & JNPT 39-01 & JNPT 762 & JNPT 749 \\
\hline JNPT 779 & JNPT 25-01 & JNPT 63-01 A & JNPT (S) $10 \mathrm{~F}$ & JNPT (S) $10 \mathrm{G}$ & JNPT 779 & JNPT 831 & JNPT 755 \\
\hline JNPT 778 & JNPT-49 & JNPT 799 & JNPT (S) $10 \mathrm{G}$ & JNPT 800 & JNPT 749 & JNPT 787 & JNPT 800 \\
\hline JNPT 777 & JNPT-25 & JNPT 35-01 & JNPT (S) 10 & JNPT (S) 7-1 B & JNPT 816 & IR64 & JNPT-23 \\
\hline JNPT 752 & JNPT 760 & JNPT 773 & JNPT 772 & JNPT 770 & JNPT 24-01 & Sahbhagi & JNPT 822 \\
\hline JNPT 811 & JNPT (S) 6-6 B & JNPT 851 & JNPT (S) 10-1 A & JNPT (S) $10 \mathrm{H}$ & JNPT 748 & JNPT 850 & JNPT 820 \\
\hline JNPT 751 & JNPT 761 & JNPT 39-01 & JNPT (S) 10-1-1 B & JNPT 38-01 & JNPT 57-01 & JNPT 807 & JNPT 753 \\
\hline JNPT 846 & JNPT-01 & JNPT (S) 10-1-1 A & JNPT (S) 10 B & JNPT 754 & JNPT 783 & JNPT 838 & JNPT 751 \\
\hline
\end{tabular}




\begin{tabular}{|c|c|c|c|c|c|c|c|}
\hline PC1 & PC2 & PC3 & PC4 & PC5 & PC6 & PC7 & PC8 \\
\hline JNPT 819 & JNPT 27-01 & JNPT 802 & JNPT (S) $10 \mathrm{H}$ & JNPT 841 & JNPT 38-01 & JNPT 80-01 & JNPT 811 \\
\hline JNPT 750 & JNPT-17 & JNPT 782 & JNPT (S) 10 D & JNPT 834 & JNPT 40-01 & JNPT 828 & JNPT 810 \\
\hline JNPT 749 & JNPT 57-01 & JNPT 25-01 & JNPT-41 & JNPT (S) 7-1 C & JNPT 764 & JNPT 776 & JNPT 752 \\
\hline JNPT 796 & JNPT 758 & JNPT 758 & JNPT 788 & $\mathrm{JRH}-8$ & HMT & JNPT 767 & JNPT (S) 8-1 A \\
\hline $\begin{array}{l}\text { JNPT (S) } \\
8-1 \text { B }\end{array}$ & JNPT 754 & JNPT (S)23-1 A & JNPT-47 & JNPT 840 & JNPT 63-01 A & JNPT 821 & JNPT 63-01 B \\
\hline JNPT 748 & JNPT 844 & JNPT 808 & JNPT-49 & JNPT 79-01 & JNPT13-01 & JNPT (S) 35-2 A & JNPT 850 \\
\hline JNPT 824 & JNPT-35-1 & JNPT 756 & JNPT (S)4-1 & JNPT (S) 7-1 A & $\begin{array}{l}\text { JNPT (S) 10-1 } \\
\text { C }\end{array}$ & JNPT 843 & JNPT 788 \\
\hline JNPT 800 & JNPT 845 & JNPT 794 & JNPT 770 & JNPT 781 & JNPT 750 & JNPT 765 & JNPT 65-01 \\
\hline JNPT 780 & JNPT 810 & JNPT 79-01 & JNPT (S) $10 \mathrm{~A}$ & JNPT (S) 35-2 A & JNPT 41-01 & JNPT 819 & JNPT 23-01 \\
\hline JNPT 820 & JNPT 38-01 & JNPT 760 & JNPT 845 & JNPT 774 & JNPT 752 & JNPT (S)23-1 & JNPT 748 \\
\hline JNPT 843 & & JNPT 791 & JNPT 848 & JNPT 830 & JNPT 777 & JNPT 847 & JNPT 757 \\
\hline JNPT 822 & & JNPT 762 & JNPT 791 & JNPT (S) 10-1-1 A & JNPT 23-01 & JNPT 761 & JNPT 825 \\
\hline JNPT 830 & & & JNPT (S) 10 C & JNPT 57-01 & JNPT 771 & JNPT 25-01 & JNPT 71-01 \\
\hline
\end{tabular}

\section{Conclusion}

Selecting the high yielding lines in rice the characters viz., panicle weight per plant, panicle index, biological yield per plant, harvest index, number of productive tiller per plant, spikelet density, panicle length, spikelet fertility and 1000 -grain weight might be considered. On the basis of PC score, JNPT 810, JNPT 754, JNPT 800, JNPT 752, JNPT 811, JNPT 751, JNPT 748, JNPT 820, JNPT 822 and JNPT 830 were the selected 10 promising lines for both yield and quality attributes.

\section{References}

Al-Jibouri, H.A., Miller, R.A., Robinson, H.F., 1958. Genotypic and environmental variance and covariance in upland cotton crosses of interspecific origin. Agronomy Journal 50, 633-637.

Ariyo, O.N., 1987. Multivariate analysis and choice of parents in Okra (A. esculentus). Annals of Applied Biology 116, 335-341.

Ashfaq, M., Khan, A.S., Khan, S.H.U., Ahmad, R., 2012. Association of various morphological traits with yield and genetic divergence in rice (Oryza sativa L.). International Journal of Agriculture and Biology 14, 55-62.

Bekele, B.D., Rakhi, S., Naveen, G.K., Kundur, P.J., Shashidhar, H.E., 2013. Estimation of genetic variability and correlation studies for grain zinc concentrations and yield related traits in selected rice (Oryza sativa L.) genotypes. Asian Journal of Experimental Biological Sciences 4(3), 345-351.

Burton, G.W., 1952. Quantitative inheritance of grasses. Proc $6^{\text {th }}$ Int. Grassland Congress crested wheat seed production. Agronomy Journal 51, 515-518.

Burton, G.W., De Vane, E.H., 1953. Estimating heritability in tall fescue (Festuca arundinacea) from replicated clonal material. Agronomy Journal 45, 478-481.

Chakravorty, A., Ghosh, P.D., Sahu, P.K., 2013. Multivariate analysis ofphenotypic diversity of landraces ofrice of West Bengal. AmericanJournal of Experimental Agriculture 3(1), 110-123.

Cheng, S., Mao, C.Z., Zhan, X.D., Si, H., Sun, Z.X., 2001. Construction of double haploid $(\mathrm{DH})$ and recombinant inbred lines (RILs) population of indica-japonica hybrid and their differential in indica and japonica property. Chinese Journal of Rice Science 15(4), 257-260.

Dewey, D.R., Lu, K.H., 1959. A correlation and path coefficient analysis of yield components of the crested wheat grass seed production. Agronomy Journal 51, 1207-1211.

Dongre, P.R., Mishra, D.K., Koutu, G.K., Singh, S.K., 2014. Estimation of genetic variability and correlation for grain yield and its components in RILs derived population of rice. JNKVV Research Journal 48(1), 55-59.

Johnson, H.W., Robinson, H.F., Comstock, R.E., 1955. Genotypic and phenotypic correlation in soybean and their implication in selection. Agronomy Journal 47, 477-483.

Jolliffe, I.T., 1986. Principal Component Analysis. Springer, New York.

Juliano, B.O., 1971. A simplified assay for milled rice amylose. Cereal Science Today 16, 334-339.

Khush, G.S., 1995. Breaking the yield frontier of rice. Geo Journal 35, 329-332.

Khush, G.S., 1999. Green revolution: preparing for the $21^{\text {st }}$ century, Genome 42, 646-655 
Kumar, N., Trwari, B., Lai, G.M., Mishra, S.P., Katiyar, A., Khuntey, Y., 2015. Evaluation of rice hybrids (Oryza sativa L.) for yield and its component characters. Indian Research Journal of Genetics \& Biotechnology 7(1), 41-43.

Kumar, V., Koshta, N., Sohgaura, N., Koutu, G.K., 2014. Genetic evaluation of rils population for yield and quality attributing traits in rice (Oryza sativa L.). Journal of Agricultural Technology 1(1), 43-51.

Massay, W.F., 1965. Principal components regression in exploratory statistical research. Journal of the American Statistical Association 60, 234-246.

Nair, N.V., Balakrishnan, R. and Sreenivasan, T.V., 1998. Variabilityof quantitative traits in exotic hybrid germplasm of sugarcane. Genetic Resources and Crop Evolution 45, 459-464.

Narciso, J., Hossain, M., 2002. In: IRRI World Rice Statistics, http://www.irri.org/science/ricestat

Nassir, A., 2002. Studies on genotypexenvironment interactions, variability and plant character association in rice. Ph. D. thesis, University of Agriculture, Abeokuta, Nigeria.

Panse, V.G., Sukhatme, P.V., 1967. Statistical methods of agricultural workers, $2^{\text {nd }}$ Edition, 381, I.C.A.R Publishing, New Delhi.

Rajput, A.S., Babu, G.S., Bhatti, M., 2014. Genetic diversity of irrigated medium duration of rice genotypes suited for eastern plain zone of U.P. IOSR Journal of Agriculture and Veterinary Science 7(7), 42-45.
Ravindrababu, V., Shreya, K., Kuldeep Singh, D., Usharani, G., Siva Shankar, A., 2012. Correlation and path analysis studies in popular rice hybrids of India. International Journal of Scientific and Research Publications 2(3), 1-5.

Shobha Rani, N., Subba Rao, L.V., Viraktamath, B.C., 2006. National Guidelines for the conduct of tests for Distinctness, Uniformity and Stability, Rice. Directorate of Rice Research, Rajendra nagar, Hyderabad.

Shrivastava, A., Mishra, D.K., Koutu, G.K., Singh, S.K., 2014. Heritability and genetic advance estimation from parental lines of hybrid rice. International journal of scientific research, 3(7), 11-13.

Singh, R.K., Chaudhary, B.D., 2005. Biometrical methods in quantitative genetic analysis. Kalyani Publishers, New Delhi.

Singh, S.K., Sharma, S., Koutu, G.K., Mishra, D.K., Singh, P., Prakash, V., Kumar, V., 2014. Genetic diversity in npt lines derived from Indica $\times$ Japonica subspecies crosses of rice using SSR markers. Scholarly Journal of Agriculture Science 4(3), 121-132.

Sohgaura, N., Mishra, D.K., Koutu, G.K., Singh, S.K., Kumar, V., 2014. Genetic evaluation of inter sub-specific derived rils population for yield and quality attributes in rice. Trends in Biosciences 7(18), 2631-2638.

Yang , X.H.,Yuan, J., Chen, H.C., He, H.Y., Chen, X.J., You, J.M., Wu, S.P., Wang, Y.Y., 2009. Principal component analysis of major agronomic traits on upland rice germplasm resources in Guizhou. Southwest China Journal of Agricultural Sciences 22(5), 1204-1208. 